\title{
Molecular Breast Imaging: Administered Activity Does Not Require Adjustment Based on Patient Size
}

\author{
Thuy D. Tran, CNMT, Lacey R. Ellingson, CNMT, Tiffinee N. Swanson, CNMT, Courtney M. Solberg, CNMT, \\ Michael K. O'Connor, PhD, and Carrie B. Hruska, PhD
}

Department of Radiology, Mayo Clinic, Rochester, Minnesota

\begin{abstract}
At our institution, molecular breast imaging (MBI) is performed with $300 \mathrm{MBq}$ of ${ }^{99 \mathrm{~m} T c}$-sestamibi for all patients. For some nuclear medicine procedures, administered activity or imaging time is increased for patients of larger size to obtain adequate
\end{abstract} counts. Our objective was to assess whether uptake of ${ }^{99 \mathrm{mT}} \mathrm{T}-$ sestamibi in the breast is influenced by patient size. Methods: Records from patients who underwent a clinical MBI examination between July and November 2016 were reviewed. Those in whom our standard injection and preparation techniques were followed were included in the analysis. Patients were injected with approximately $300 \mathrm{MBq}$ of ${ }^{99 \mathrm{~m} T c-s e s t a m i b i . ~ R e s i d u a l ~ a c-~}$ tivity was measured to allow calculation of exact administered activity for each patient. Breast images were acquired at $10 \mathrm{~min} /$ view using a dual-head cadmium zinc telluride-based $y$-camera. Breast thickness was measured as the distance between the 2 detectors. Patient height, weight, body surface area, and body mass index were obtained from records. Lean body mass with the James equation ( LBM $\left._{\text {James }}\right)$ and Janmahasatian correction ( LBM $_{\text {Janma }}$ ) was calculated. Count density in the breast tissue was measured by drawing a region of interest around the central breast tissue of the right breast mediolateral-oblique view of the lower detector. Count density was expressed as $\mathrm{cts} / \mathrm{cm}^{2} / \mathrm{MBq}$ of administered activity. Spearman correlation coefficient $\left(r_{s}\right)$ was calculated. Results: A total of 200 patients were analyzed. No dose infiltration was suspected at any injection. Average administered activity was $292 \mathrm{MBq}$ (SD, 13.8 MBq; range, 247-326 MBq). Average count density was $7.2 \mathrm{cts} / \mathrm{cm}^{2} / \mathrm{MBq}\left(\mathrm{SD}, 2.7 \mathrm{cts} / \mathrm{cm}^{2} / \mathrm{MBq}\right.$; range, $\left.3.1-17.8 \mathrm{cts} / \mathrm{cm}^{2} / \mathrm{MBq}\right)$. MBI count density was weakly negatively correlated with height $\left(r_{\mathrm{s}}=-0.18 ; P=0.01\right)$, weight $\left(\mathrm{r}_{\mathrm{s}}=\right.$ $-0.23 ; p=<0.001)$, body mass index $\left(r_{\mathrm{s}}=-0.16 ; P=0.02\right)$, body surface area $\left(r_{\mathrm{s}}=-0.22 ; P=0.002\right), \mathrm{LBM}_{\text {James }}\left(\mathrm{r}_{\mathrm{s}}=\right.$ -0.23; $P=0.001)$, and LBM Janma $\left(r_{\mathrm{s}}=-0.23 ; P=0.001\right)$. No correlation was observed between count density and breast thickness $\left(r_{s}=0.06 ; P=0.37\right)$. Conclusion: Our results suggest a lack of relationship between uptake of ${ }^{99 \mathrm{mT}} \mathrm{Tc}$-sestamibi in breast tissue and body size or compressed breast thickness. Altering from the standard $300 \mathrm{MBq}$ of administered activity for larger patients is likely unnecessary.

Key Words: breast; molecular imaging; oncology; breast; breast count density; dose regimen; molecular breast imaging; ${ }^{99 m T c-}$ sestamibi; body mass index

Received Nov. 13, 2017; revision accepted Jan. 4, 2018.

For correspondence or reprints contact: Carrie B. Hruska, Department of

Radiology, Mayo Clinic, 200 First St. SW, Rochester, MN 55905-0001.

E-mail: hruska.carrie@mayo.edu

Published online Mar. 29, 2018.

COPYRIGHT @ 2018 by the Society of Nuclear Medicine and Molecular Imaging.
J Nucl Med Technol 2018; 46:265-267

DOI: $10.2967 / \mathrm{jnmt} .117 .203869$

$\mathbf{M}$ olecular breast imaging (MBI) is a low-dose nuclear medicine test that uses dedicated semiconductor-based $\gamma$-cameras and injection of a radiotracer, primarily ${ }^{99 \mathrm{~m}} \mathrm{Tc}-$ sestamibi, to examine functional behavior of breast tissue (1). MBI has shown utility as a supplemental screening tool for detecting mammographically occult breast cancers in women with dense breast tissue (1).

In the last few years, efforts to standardize MBI examinations have focused on several factors that influence uptake of ${ }^{99 \mathrm{~m} T c-s e s t a m i b i}$ in the breast tissue. In one study, patients were asked to exercise on a treadmill during injection; this practice was found to have the undesired effect of decreasing sestamibi uptake in the breast tissue (2). A study of the effects of caffeine intake showed it had no effect on sestamibi uptake (2). However, the combination of the following 3 methods was shown to increase sestamibi uptake in the breast tissue by approximately 50\%: patient fasting before injection, placing a warm blanket around the patient before injection, and using a syringe known to provide minimum residual activity for dose injections $(2,3)$. Although these measures were found to help improve uptake in the breast tissue, count density was still highly variable among patients (2), suggesting that further investigation of other factors influencing sestamibi uptake in the breast tissue is warranted.

Because fasting, warming, and using low-adhesion syringes are now part of our standardized MBI practice, a closer look at the impact of patient size on ${ }^{99 \mathrm{~m}} \mathrm{Tc}$-sestamibi uptake in the breast tissue is necessary. For some nuclear medicine procedures, administered activity or imaging time is increased for patients of larger body habitus to obtain adequate image counts. For example, according to the American Society of Nuclear Cardiology Stress Protocol Guidelines, increased administered activity should be considered for patients weighing over $113 \mathrm{~kg}$ undergoing myocardial perfusion imaging to achieve sufficient count statistics in image quality (4). The purpose of this study was to evaluate the effects of patient size on sestamibi 
FIGURE 1. Region of interest of right mediolateraloblique view of lower detector.

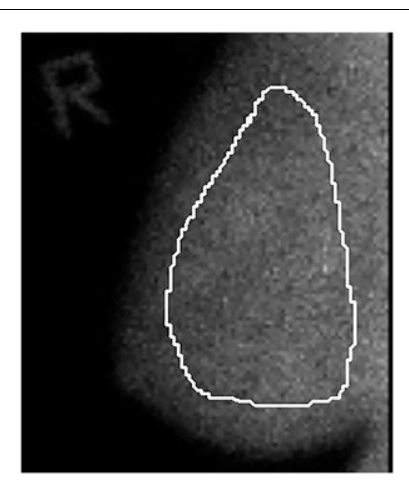

uptake in breast tissue under optimal patient preparation and determine whether weight-based administered activity should be used in MBI examinations.

\section{MATERIALS AND METHODS}

Retrospective analysis of patient records was conducted under a minimum-risk Institutional Review Board-approved and
Health Insurance Portability and Accountability Act-compliant research protocol. Records from patients who underwent a clinical MBI examination between July and November 2016 were reviewed. Patients who followed the standard injection and preparation techniques as described below were included in the analysis (2,3). Patients with breast implants were excluded from this analysis.

MBI examinations were performed using a dedicated dualhead cadmium zinc telluride-based $\gamma$-camera optimized for lowdose imaging (LumaGem; $\gamma$-Medica) (5). Before the injection of ${ }^{99 m}$ Tc-sestamibi, patients fasted for a minimum of $3 \mathrm{~h}$. A warm blanket was placed around the patient's shoulders and chest for approximately $5 \mathrm{~min}$ before injection to facilitate peripheral blood flow and increase uptake of ${ }^{99 \mathrm{~m}} \mathrm{Tc}$-sestamibi in the breast tissue (2). After explanation of examination, patients were injected with approximately $300 \mathrm{MBq}$ of ${ }^{99 \mathrm{~m}} \mathrm{Tc}$-sestamibi using a syringe known to provide low adhesion of sestamibi (3). The radiotracer was administered intravenously to the patient's arm or hand using a butterfly needle or indwelling catheter followed by a $10-\mathrm{mL}$ saline flush. Preinjection activity, time of injection, and postinjection residual activity were measured to allow calculation of exact administered activity for each patient.

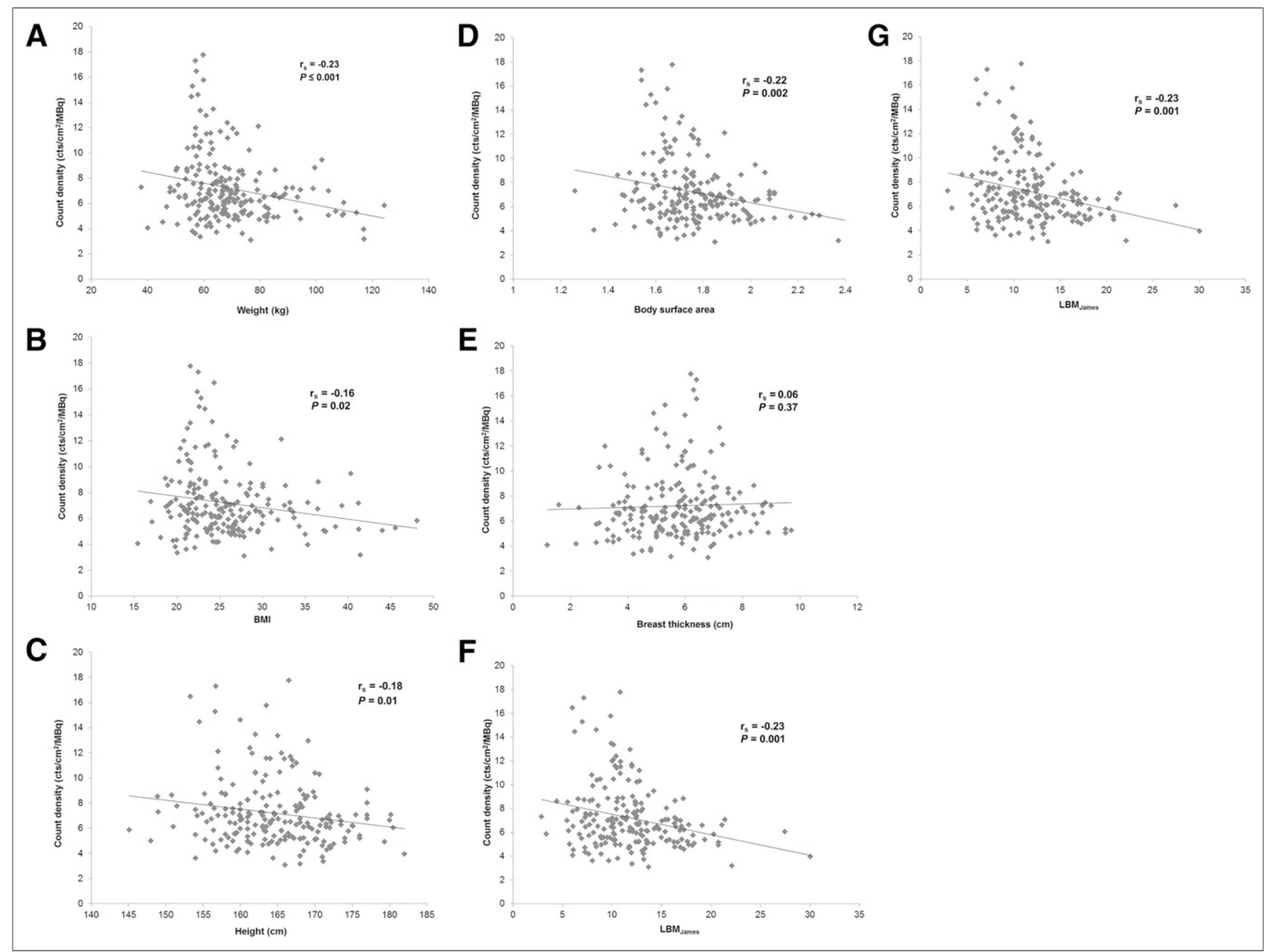

FIGURE 2. Relationship between count density and patient size factors. Scatterplot shows relationship between count density and patient weight $(A)$, patient body mass index (BMI) $(B)$, height $(C)$, body surface area (D), breast thickness (E), and lean body mass ( $F$ and $G$ ). 
TABLE 1

Correlation Between Count Density and Patient Characteristics

\begin{tabular}{lcc}
\hline Patient characteristic & $r_{\mathrm{s}}$ & $P$ \\
\hline Weight & -0.23 & $<0.001$ \\
Height & -0.18 & 0.01 \\
Body mass index & -0.16 & 0.02 \\
Body surface area & -0.22 & 0.002 \\
LBM James $_{\text {LBM Janma }}$ & -0.23 & 0.001 \\
Breast thickness & -0.23 & 0.001 \\
\hline
\end{tabular}

Spearman $\rho\left(r_{s}\right)=<0.19$ (very weak), 0.20-0.39 (weak), 0.400.59 (moderate), $0.60-0.79$ (strong), and 0.80-1.0 (very strong).

Patient positioning and imaging began immediately after injection. Bilateral craniocaudal and mediolateral-oblique views were acquired for 10 min per view using standard mammographic positioning techniques. Light compression was applied to the breast to limit patient motion, and breast thickness was recorded as the distance between the 2 detectors. Patient height, weight, body surface area, and body mass index were extracted from records closest to the date of MBI examination (median time from MBI, $2 \mathrm{~d}$; interquartile range, 0-53 d). In addition, lean body mass was calculated using the James $\left(\mathrm{LBM}_{\mathrm{James}}\right)$ and Janmahasatian $\left(\mathrm{LBM}_{\text {Janma }}\right)$ equations specific to women in estimating patient's body weight minus adipose tissue (6).

The right breast mediolateral-oblique view of the lower detector for each patient was used for count density analysis. The count density in the breast tissue was measured by drawing a region of interest around the central breast tissue excluding the pectoral muscle, any lesions, and edges of the detector, using in-house image analysis software (Fig. 1). Count density was expressed as $\mathrm{cts} / \mathrm{cm}^{2} / \mathrm{MBq}$ of administered activity. The relationship between count density and patient body size factors was assessed with Spearman correlation coefficient $\left(\mathrm{r}_{\mathrm{s}}\right)$. A $P$ value of less than 0.05 was considered significant.

\section{RESULTS}

Two hundred patients were analyzed. No dose infiltration was suspected at any injection. Average age of the study group was 57 y (SD, 9.7 y; range, 35-86 y). The average administered activity, calculated after accounting for residual activity, was $292 \mathrm{MBq}$ (SD, $13.8 \mathrm{MBq}$; range, 247-326 $\mathrm{MBq})$. The average count density on MBI was $7.2 \mathrm{cts} / \mathrm{cm}^{2} /$ $\mathrm{MBq}$ (SD, $2.7 \mathrm{cts} / \mathrm{cm}^{2} / \mathrm{MBq}$; range, $3.1-17.8 \mathrm{cts} / \mathrm{cm}^{2} / \mathrm{MBq}$ ).

As given in Table 1 and shown in Figure 2, MBI count density was weakly negatively correlated with height, weight, body mass index, body surface area, $\mathrm{LBM}_{\mathrm{James}}$, and $\mathrm{LBM}_{\mathrm{Janma}}$ (all $P<0.02$ ). No correlation was observed between count density and breast thickness $(P=0.37)$.

\section{DISCUSSION}

The results of this study reveal that patient size characteristics do not significantly influence uptake of ${ }^{99 \mathrm{~m}} \mathrm{Tc}$-sestamibi in breast tissue. Despite all patients following standardized patient preparation and injection procedures, we did not observe a consistent relationship between count density and patient size factors based on height and weight or breast thickness. These findings are in contrast to other nuclear medicine publications indicating a weight-based dose regimen to be beneficial in improving image quality $(4,7)$. A wide range of count densities on MBI was observed in this cohort $\left(3.1-17.8 \mathrm{cts} / \mathrm{cm}^{2} / \mathrm{MBq}\right)$, suggesting that other factors not accounted for in this study (i.e., liver function or inherent mitochondrial activity of breast tissue) influence sestamibi uptake in breast tissue.

There were some limitations in the design of this study. The patient's fasting status at the time of injection was patientreported and not verified through quantitative means such as glucose testing. It is possible that some patients may not have strictly followed fasting instructions, which may influence the uptake in breast tissue. Also, as shown in a previous study, light to moderate exercise performed at the time of injection decreases sestamibi uptake in the breast tissue as uptake is increased in muscle tissue elsewhere in the body. Patients were not asked if they had completed an exercise workout before their MBI examination. It is possible that the residual effects of a workout could affect the distribution of sestamibi in the body. However, these results reflect the findings that would be observed in a typical clinical practice setting.

\section{CONCLUSION}

Our results showed either a weak or a lack of relationship between uptake of ${ }^{99 \mathrm{~m}} \mathrm{Tc}$-sestamibi in breast tissue and patient size factors including height, weight, body mass index, body surface area, lean body mass, and compressed breast thickness. Altering from the standard $300 \mathrm{MBq}$ of administered activity based on patient size is likely unnecessary for MBI examinations.

\section{DISCLOSURE}

This research was funded in part by grants from the Mayo Foundation and Mayo Center for Individualized Medicine. No other potential conflict of interest relevant to this article was reported.

\section{REFERENCES}

1. Hruska CB. Molecular breast imaging for screening in dense breasts: state of the art and future directions. AJR. 2017;208:275-283.

2. O'Connor MK, Hruska CB, Tran TD, et al. Factors influencing the uptake of 99m Tc-sestamibi in breast tissue on molecular breast imaging. J Nucl Med Technol. 2015;43:13-20.

3. Swanson TN, Troung DT, Paulsen A, Hruska CB, O'Connor MK. Adsorption of ${ }^{99 \mathrm{~m}} \mathrm{Tc}$ sestamibi onto plastic syringes: evaluation of factors affecting the degree of adsorption and their impact on clinical studies. J Nucl Med Technol. 2013;41:247-252.

4. Henzlova MJ, Duvall WL, Einstein AJ, Travin MI, Verberne HJ. ASNC imaging guidelines for SPECT nuclear cardiology procedures: stress, protocols, and tracers. J Nucl Cardiol. 2016;23:640-642.

5. Hruska CB, Weinmann AL, O'Connor MK. Proof of concept for low-dose molecular breast imaging with a dual-head CZT gamma camera: part I-evaluation in phantoms. Med Phys. 2012;39:3466-3475.

6. Tahari AK, Chien D, Azadi JR, Wahl RL. Optimum lean body formulation for correction of standardized uptake value in PET imaging. J Nucl Med. 2014;55:1481-1484.

7. Ghanem MA, Kazim NA, Elgazzar AH. Impact of obesity on nuclear medicine imaging. J Nucl Med Technol. 2011;39:40-50. 